\title{
ZONOIDS WHOSE POLARS ARE ZONOIDS
}

\author{
ROLF SCHNEIDER
}

ABSTRACT. It is shown that Euclidean $n$-space contains nonellipsoidal, centrally symmetric convex bodies which, as well as their polars, are zonoids (i.e., can be approximated by finite sums of segments). This disproves a conjecture of E. D. Bolker.

A zonoid in Euclidean $n$-space $\mathbf{R}^{n}$ is a convex body which can be approximated (with respect to the Hausdorff metric) by finite vector sums of line-segments. There are several equivalent definitions of zonoids (e.g., as ranges of nonatomic vector measures) for which the reader is referred to Bolker [1]. In [1, p. 340], and again in [2], Bolker conjectured that the only zonoids in $\mathbf{R}^{n}(n \geq 3)$, whose polar bodies (taken with respect to the centre) are also zonoids, are the ellipsoids. Equivalently this would mean (compare $[1, \mathrm{p} .340])$ that the Euclidean space $\mathbf{R}^{n}(n \geq 3)$ is the only $n$-dimensional Banach space which is isometric to a subspace of $L^{1}$ and to a quotient space of $L^{\infty}$. However, Bolker's conjecture is untrue as we shall now show. Let

$$
S=\left\{x \in \mathbf{R}^{n}:\langle x, x\rangle=1\right\}
$$

denote the unit sphere of $\mathbf{R}^{n}$, and let

$$
B=\left\{x \in \mathbf{R}^{n}:\langle x, x\rangle \leq 1\right\}
$$

be its unit ball $\left(\langle\right.$,$\left.\rangle is the scalar product of \mathbf{R}^{n}\right)$. For a real function $F$ on $S$ for which it makes sense we denote the sup-norm by $\|F\|$ and the $L^{2}$-norm with respect to Lebesgue measure $\omega$ on $S$ by $\|F\|_{L}$. For $F$ of class $C^{2 r}$ let

$$
\|F\|_{r}=a_{n}\|F\|_{L}+b_{n}\left\|\Delta^{r} F\right\|_{L}
$$

where $\Delta$ denotes the Laplace operator on $S$ and where $a_{n}, b_{n}$ are positive

Received by the editors February 8, 1974.

AMS (MOS) subject classifications (1970). Primary 52A20; Secondary 46B99, 28A45.

Key words and phrases. Zonoid, range of vector measure, polar convex bodies, isometries of finite-dimensional subspaces of $L^{1}$ and quotient spaces of $L^{\infty}$. 
constants which will be defined later. The support function $H_{K}$ (restricted to $S$ ) of a convex body $K \subset \mathbf{R}^{n}$ is defined by

$$
H_{K}(y)=\sup \{\langle x, y\rangle: x \in K\}
$$

for $y \in S$. By $\mathbb{S}_{n}$ we denote the set of all convex bodies in $\mathbf{R}^{n}$ which are centrally symmetric with respect to the origin and whose support function is of class $C^{2 r}$, where $2 r=n+2$ or $n+3$, according to whether $n$ is even or odd. On $\mathcal{S}_{n}$ we define a metric $d$ by $d(K, L)=\left\|H_{K}-H_{L}\right\|_{r}$.

Proposition. In $\left(\mathbb{S}_{n}, d\right)$, a whole neighborhood of $B$ consists of zonoids.

Proof. We shall use the fact that the convex body $K$ is a zonoid if there exists a positive Borel measure $\mu$ on $S$ such that $H_{K}(y)=$ $\int|\langle x, y\rangle| d \mu(x), y \in S$ (all integrations are over $S$ ). For $K \in \mathcal{S}_{n}$ there exists a uniquely determined even, continuous function $h_{K}$ on $S$ for which

$$
H_{K}(y)=\int|\langle x, y\rangle| b_{K}(x) d \omega(x), \quad y \in S
$$

see [4]. A slight extension of the argument used in [4] will yield a bound for $\left\|h_{K}\right\|$ in terms of $\left\|H_{K}\right\|_{r}$.

For real continuous functions $F, G$ on $S$ we define the inner product by

$$
(F, G)=\int F G d \omega
$$

For $m=0,1,2, \cdots$ let $\left\{Y_{m j}: j=1, \cdots, N(n, m)\right\}$ be an orthonormal basis of the space of spherical surface harmonics of degree $m$ on $S$. Using the addition theorem for spherical harmonics (Müller [3, p. 10]), the CauchySchwarz inequality, and the equality (22) of [3, p. 15], we arrive at

$$
\left|\sum_{j=1}^{N(n, m)}\left(F, Y_{m j}\right) Y_{m j}(x)\right| \leq\left[\frac{N(n, m)}{\omega_{n}}\right]^{1 / 2}\|F\|_{L}, \quad x \in S,
$$

where $\omega_{n}=\omega(S)$.

If $K \in \mathbb{S}_{n}$, the function $h_{K}$ which satisfies (1) is given by

$$
b_{K}(x)=\sum_{m=0 ; 2 \mid m}^{\infty} \lambda_{m}^{-1} \sum_{j=1}^{N(n, m)}\left(H_{K}, Y_{m j}\right) Y_{m j}(x), \quad x \in S,
$$

where 


$$
\lambda_{m}=\frac{(-1)^{1 / 2(m-2)} \pi^{1 / 2(n-1)} \Gamma(m-1)}{2^{m-2} \Gamma(1 / 2 m) \Gamma(1 / 2(m+n+1))}
$$

see [4]. As noticed in [4], repeated application of Green's formula leads to

$$
\left(H_{K}, Y_{m j}\right)=[-1 / m(m+n-2)]^{r}\left(\Delta^{r} H_{K}, Y_{m j}\right) \text {, }
$$

$$
j=1, \cdots, N(n, m), \quad m=1,2, \cdots .
$$

From (3), (4), and (2) it follows that

$$
\begin{array}{r}
\left|b_{K}(x)\right| \leq\left|\lambda_{0}^{-1}\right| \omega_{n}^{-1 / 2}\left\|H_{K}\right\|_{L}+\sum_{m=2 ; 2 \mid m}^{\infty}\left|\lambda_{m}^{-1}\right| m^{-2 r}\left[\frac{N(n, m)}{\omega_{n}}\right]^{1 / 2}\left\|\Delta^{r} H_{K}\right\|_{L} \\
x \in S
\end{array}
$$

If we now assume that $a_{n}, b_{n}$ have been defined by

$$
a_{n}=\left|\lambda_{0}^{-1}\right| \omega_{n}^{-1 / 2}, \quad b_{n}=\sum_{m=2 ; 2 \mid m}^{\infty}\left|\lambda_{m}^{-1}\right| m^{-2 r}\left[\frac{N(n, m)}{\omega_{n}}\right]^{1 / 2}
$$

(see [4] for the convergence of the latter series), then we have $\left\|h_{K}\right\| \leq\left\|H_{K}\right\|_{r}$. Clearly the function $h_{B}$ is a positive constant. Hence if $K \in \widehat{C}_{n}$ and $d(K, B) \leq h_{B}$, then

$$
\left\|b_{K}-b_{B}\right\| \leq\left\|H_{K}-H_{B}\right\|_{r}=d(K, B) \leq b_{B},
$$

which implies that $h_{K}$ is nonnegative and, therefore, that $K$ is a zonoid. This proves the Proposition.

It is now easy to construct nonellipsoidal zonoids in $\mathbf{R}^{n}$ whose polars are also zonoids. Choose an even real function $f$ on $S$ of class $C^{\infty}$ which does not vanish identically, and put $x_{\lambda}(u)=[1+\lambda f(u)] u$ for $u \in S$ and $\lambda>0$. For $\lambda$ sufficiently small, the map $x_{\lambda}: S \rightarrow \mathbf{R}^{n}$ defines the boundary of a centrally symmetric convex body $K_{\lambda}$. Let $e_{\lambda}(u)$ denote the exterior unit normal vector to $x_{\lambda}(S)$ at $x_{\lambda}(u)$. For $\lambda$ sufficiently small, the map $e_{\lambda}: S \rightarrow S$ is a diffeomorphism; let $e_{\lambda}^{-1}$ denote the inverse diffeomorphism. Then $H_{\lambda}(v)=\left\langle x_{\lambda}\left(e_{\lambda}^{-1}(v)\right), v\right\rangle, v \in S$, is the support function of the body $K_{\lambda}$, while the support function of its polar $K_{\lambda}^{*}$ is simply given by $H_{\lambda}^{*}(u)=[1+\lambda f(u)]^{-1}$, $u \in S$. It is now a matter of standard calculations to show that $\lambda>0$ can be chosen so small that $K_{\lambda}, K_{\lambda}^{*} \in \mathcal{S}_{n}$ and that $\left\|H_{\lambda}-H_{B}\right\|_{r} \leq h_{B}$ and $\left\|H_{\lambda}^{*}-H_{B}\right\|_{r} \leq h_{B}$, which implies that both $K_{\lambda}$ and $K_{\lambda}^{*}$ are zonoids. 


\section{REFERENCES}

1. E. D. Bolker, A class of convex bodies, Trans. Amer. Math. Soc. 145 (1969), 323-345. MR 41 \#921.

2. - The zonoid problem, Amer. Math. Monthly 78 (1971), 529-531.

3. C. Müller, Spherical harmonics, Lecture Notes in Math., vol. 17, SpringerVerlag, Berlin and New York, 1966. MR 33 \#7593.

4. R. Schneider, $Z u$ einem Problem von Shephard über die Projektionen konvexer Körper, Math. Z. 101 (1967), 71-82. MR 36 \#2059.

FACHBEREICH MATHEMATIK, TECHNISCHE UNIVERSITÄT BERLIN, BERLIN, GERMANY

Current address: Mathematisches Institut der Universität, 7800 Freiburg i. Br., Hebelstrasse 40, Germany 